\title{
The Existence of Periodic Solutions for Planar Multi- parameter Autonomous Perturbed Systems
}

\author{
Zhimin Luo \\ Department of Education Luoding Polytechnic Luoding 527200, Guangdong, China
}

\begin{abstract}
This paper studies the existence of periodic solutions of autonomous multi- parameter perturbation system by using Liapunov second method when the critical point of the plane autonomous system is the real center. We obtain a necessary condition and a sufficient condition which extend those in references. An example is provided to illustrate the application of the results.
\end{abstract}

Keywords-differential equation; perturbation; periodic solution; Liapunov second method

\section{INTRODUCTION}

Many physical, biological and economic problems can be attributed to the differential equation model with parameters. In general, when the parameter value changes, the motion properties of the differential system will change. Only when the parameter is sufficiently small, it is possible to keep the properties of the original motion. In particular, the emergence and disappearance of periodic solutions is a problem that has been paid attention to in the bifurcation theory of dynamical systems and attracted the attention of many scholars(see. references etc.[1,2,3,4,5,6,7]) . It is common to discuss the periodic solutions of systems with one parameter or two parameters, but there are few studies on the periodic solutions of the systems with multiple parameters. This paper discusses the following autonomous perturbed systems with multiple parameters

$$
\left\{\begin{array}{l}
\dot{x}=P(x, y)+\sum_{i=1}^{m} \lambda_{i} f_{i}(x, y) \\
\dot{y}=Q(x, y)+\sum_{i=1}^{m} \lambda_{i} h_{i}(x, y)
\end{array}\right.
$$

Where $1 \leq m<+\infty, \lambda_{i} \neq 0(i=1,2 \ldots m)$. There exists a first order continuous partial derivative for the function $P, Q, f_{i}$ and $h_{i}$. If $\lambda_{i}=0$, the system(1) can be regarded as the

$$
\left\{\begin{array}{l}
\dot{x}=P(x, y) \\
\dot{y}=Q(x, y)
\end{array}\right.
$$

Therefore the system(1) can be viewed as the system(2) after the $\mathrm{m}$ parameter perturbation. Suppose the point
$O(0,0)$ is a unique critical point of the system(1), and is the real center. We set up an appropriate plane rectangular coordinate system, and take a point $A(0, a)$ in the y positive half axis. Now, it is a given fact that there is a $T(a)$-periodic orbit $\Gamma_{A}$ through the point $A$ for the system(2). Assume the equations of $\Gamma_{A}$ are

$$
\left\{\begin{array}{l}
x=\varphi(t, a) \\
y=\psi(t, a)
\end{array}\right.
$$

Let the point $O(0,0)$ is still the critical point of the system (1), but not the center. At the same time, let $F(x, y)=C$ is the first integrals of the system(2), and define $R_{i}(a)$

$$
\begin{gathered}
R_{i}(a)=\int_{0}^{T(a)}\left(\frac{\partial F(\varphi(t, a), \psi(t, a))}{\partial x} f_{i}(\varphi(t, a), \psi(t, a))\right. \\
\left.+\frac{\partial F(\varphi(t, a), \psi(t, a))}{\partial y} h_{i}(\varphi(t, a), \psi(t, a))\right) d t
\end{gathered}
$$

\section{MAIN RESULtS}

THEOREM 1: Let $\lambda^{*}=\max _{i=1,2 \ldots m}\left\{\left|\lambda_{i}\right|\right\}$ is sufficiently small. For the system (1), If there is a closed orbit near the $\Gamma_{A}$, then $R_{i}(a)=0$.

Proof: Using the system(1), we find the orbital derivative the function $F(x, y)$

$$
\begin{aligned}
\left.\frac{d F}{d t}\right|_{(1)}=F_{x} \dot{x}+F_{y} \dot{y} & =F_{x}\left(P+\sum \lambda_{i} f_{i}\right) \\
& +F_{y}\left(Q+\sum \lambda_{i} h_{i}\right)
\end{aligned}
$$

Because $F(x, y)=C$ is the first integrals of the system(2), we obtain the 


$$
F_{x} P+F_{y} Q=0
$$

According to (4) and (5), we have

$$
\left.\frac{d F}{d t}\right|_{(1)}=\sum_{i=1}^{m} \lambda_{i}\left(F_{x} f_{i}+F_{y} h_{i}\right)
$$

Take the non tangent line segment $L$ (full long) containing point $A$ in the y positive half axis. It is clear that the $L$ is the non tangent line segment of the vector field $(P, Q)$. For the system(2), the trajectory starting from A will return to A after $T(a)$. When $t=0$, for the system(1) the trajectory starting from $\mathrm{A}$ is

$$
\left\{\begin{array}{l}
x=\varphi_{\lambda_{1} \lambda_{2} \ldots \lambda_{m}}(t, a) \\
y=\psi_{\lambda_{1} \lambda_{2} \ldots \lambda_{m}}(t, a)
\end{array}\right.
$$

According to the continuous dependence of solutions on initial data and parameters, if the $\lambda^{*}$ is sufficiently small, then the system(1) trajectory starting from A will return to the $y$ positive half axis after $T_{\lambda_{1} \lambda_{2} \ldots \lambda_{m}}(a)$. When the first intersection occur about the trajectory and the y positive half axis, the increase of the function $F(x, y)$ is

$$
\begin{aligned}
\Delta F_{1} & =F\left(0, \psi_{\lambda_{1} \lambda_{2} \ldots \lambda_{m}}\left(T_{\lambda_{1} \lambda_{2} \ldots \lambda_{m}}(a), a\right)\right)-F(0, a) \\
& =\left.\int_{0}^{T_{\lambda_{1} \lambda_{2} \ldots \lambda_{m}}(a)} \frac{d F}{d t}\right|_{(1)} d t \\
& =\int_{0}^{T_{\lambda_{1} \lambda_{2} \ldots \lambda_{m}}(a)} \sum_{i=1}^{m} \lambda_{i}\left(F_{x} f_{i}+F_{y} h_{i}\right) d t \\
& =\sum_{i=1}^{m} \lambda_{i} \int_{0}^{T_{\lambda_{1} \lambda_{2} \ldots \lambda_{m}}(a)}\left(F_{x} f_{i}+F_{y} h_{i}\right) d t
\end{aligned}
$$

Let

$$
\begin{aligned}
R_{i}^{*}(a)= & \int_{0}^{T_{\lambda_{1} \lambda_{2} \ldots \lambda_{m}}(a)}\left(\frac{\partial F\left(\varphi_{\lambda_{1} \lambda_{2} \ldots \lambda_{m}}(t, a), \psi_{\lambda_{1} \lambda_{2} \ldots \lambda_{m}}(t, a)\right)}{\partial X} .\right. \\
& f_{i}\left(\varphi_{\lambda_{1} \lambda_{2} \ldots \lambda_{m}}(t, a), \psi_{\lambda_{1} \lambda_{2} \ldots \lambda_{m}}(t, a)\right) \\
+ & \frac{\partial F\left(\varphi_{\lambda_{1} \lambda_{2} \ldots \lambda_{m}}(t, a), \psi_{\lambda_{1} \lambda_{2} \ldots \lambda_{m}}(t, a)\right)}{\partial y} \\
& \left.h_{i}\left(\varphi_{\lambda_{1} \lambda_{2} \ldots \lambda_{m}}(t, a), \psi_{\lambda_{1} \lambda_{2} \ldots \lambda_{m}}(t, a)\right)\right) d t
\end{aligned}
$$

Assume $a$ is the parameter. By According to the continuous dependence of solutions on initial data, taking a arbitrarily $\varepsilon>0$, there is $a_{1}>0, \delta>0$ and $\lambda_{0}>0$, so that when $\left|a_{1}-a\right|<\delta, \lambda^{*}<\lambda_{0}$,we obtain

$$
\begin{gathered}
\left|\varphi_{\lambda_{1} \lambda_{2} \ldots \lambda_{m}}\left(t, a_{1}\right)-\varphi(t, a)\right|<\varepsilon \\
\left|\psi_{\lambda_{1} \lambda_{2} \ldots \lambda_{m}}\left(t, a_{1}\right)-\psi(t, a)\right|<\varepsilon \\
\left|T_{\lambda_{1} \lambda_{2} \ldots \lambda_{m}}\left(a_{1}\right)-T(a)\right|<\varepsilon
\end{gathered}
$$

Where $t \in[0,2 T(a)]$. On the other hand, the function $F_{x} f_{i}+F_{y} h_{i}$ is continuous. When the $\lambda^{*}$ is sufficiently small and $a_{1}$ is close fully to $a$, we $R_{i}^{*}\left(a_{1}\right)$ is close fully to $R_{i}(a)$. Let $R(a)=\sum_{i=1}^{m} R_{i}(a)$ and $R^{*}(a)=\sum_{i=1}^{m} R_{i}^{*}(a)$, we obtain also that $R^{*}(a)$ is close fully to $R(a)$. If $R_{i}(a) \neq 0$, then $R_{i}^{*}\left(a_{1}\right) \neq 0$. That is when $0<\lambda^{*}<\delta$, the system(1) trajectory starting from A can not be closed orbit. The proof of theorem 1 has been complete.

THEOREM 2: Consider $a_{0}>0, R\left(a_{0}\right)=0, R(a)$ is smooth near $a_{0}$ and $R^{\prime}\left(a_{0}\right) \neq 0$.Then, ,there exists a closed orbit of the system(1) near the $\Gamma_{A}$ when the $\lambda^{*}$ is sufficiently small.

Proof: Owing to $R\left(a_{0}\right)=0, R^{\prime}\left(a_{0}\right) \neq 0, \varphi(t, a)$ and $\psi(t, a)$ are smooth, $R(a)$ can not reach to the extreme value. There is $\delta_{1}, \delta>0$ for arbitrarily $\varepsilon>0$, we obtain $R\left(a_{0}+\delta_{1}\right) \cdot R\left(a_{0}-\delta_{1}\right)<0$ as long as $0<\delta_{1}<\delta<\varepsilon$. By the continuous dependence of solutions on parameters, if $\lambda^{*}<\lambda_{0}$, then $R^{*}\left(a_{0}+\delta_{1}\right) \cdot R^{*}\left(a_{0}-\delta_{1}\right)<0$. According to the continuous dependence of solutions on initial data and the intermediate value theorem of continuous function, there exists $a_{1} \in\left(a_{0}-\delta_{1}, a_{0}+\delta_{1}\right) \subset\left(a_{0}-\varepsilon, a_{0}+\varepsilon\right) \quad$ we get $R^{*}\left(a_{1}\right)=0$. That means the system(1) orbit starting from the point $\left(0, a_{1}\right)$ in the interval $\left(a_{0}-\varepsilon, a_{0}+\varepsilon\right)$ of the $\mathrm{y}$ axis is the $T_{\lambda_{1} \lambda_{2} \ldots \lambda_{m}}\left(a_{1}\right)$ - periodic orbit. If $\delta_{1}$ is sufficiently small, According to the continuous dependence of solutions on initial data and parameters, the periodic orbit must be located within the neighborhood of $\Gamma_{A}$. The proof of theorem 2 has been complete.

\section{EXAMPLE}

Consider the next system(9), where $i=1$, 


$$
\left\{\begin{array}{l}
\frac{d x}{d t}=-y(1+x)+\lambda x(1+x)(1+y) \\
\frac{d y}{d t}=x(1+y)-\lambda y(1+x)(1+y)\left(x^{2}+\frac{y^{2}}{3}\right)
\end{array}\right.
$$

Let $\lambda=0$, the first integrals of the system(9) is

$$
F(x, y)=x-\ln (1+x)+y-\ln (1+y)=C
$$

The determining functions of periodic solution is

$$
R(a)=\int_{0}^{T(a)}\left(x^{2}(1+y)-y^{2}(1+x)\left(x^{2}+\frac{y^{2}}{3}\right)\right) d t
$$

Convert the expression (11) into a curve integral

$$
R(a)=\oint_{\Gamma(a)} x d y+y\left(x^{2}+\frac{y^{2}}{3}\right) d x
$$

The integral curve $\Gamma(a)$ is $F(x, y)=F(0, a)$, and its direction is counterclockwise. By the Green formula, we obtain

$$
R(a)=\iint_{S(a)}\left(1-\left(x^{2}+y^{2}\right)\right) d x d y
$$

Where $S(a)$ is the area enclosed by $\Gamma(a)$, assuming $R\left(a^{*}\right)=0$ and $a$ is sufficiently small, it is clear that $R(a)>0$ no matter what $a$ is big or small. So we obtain $R^{\prime}\left(a^{*}\right) \neq 0$. Accordingly the system(9) have a periodic solution when $\lambda$ is sufficiently small.

\section{REFERENCES}

[1] W.D.sheng; L. Bing. On deciding method for two-dimension containing the existence of closed orbits in the two-parameter perturbing autonomous system[J]. Bulletin of science and technology, 2012,28(11):27-29.

[2] Y.Feng; F.Gao; Z.Wang; R.Chen. Existence and uniqueness of solutions for periodically perturbed conservative systems[J]. Journal of Sichuan Normal University, 2011, 34(34):651-654.

[3] S.G .Peng; S.M.Zhu. Existence and uniqueness of periodic solutions for periodically perturbed non-conservitive systems[J]. Chinese Annals of Mathematics Series A, 2003, 3(3):293-298.

[4] Hartman P. Ordinary Differential Equations[M]. Birkhauser Boston,1982.

[5] T.Yoshizawa. Stability theory by Liapunuv's second method[M]. The Math. Soc. Of Japan, Tokyo, 1966.

[6] R.Pisani; M.Tucci. Existence of infinitely many periodic solutions for a perturbed Hamiltonian system[J]. Nonlinear Analysis Theory Methods \& Applications, 1984, 8(8):873-891.
[7] M.Kamenskii; O.Makarenkov; P.Nistri. Periodic solutions of periodically perturbed planar autonomous systems: A topological approach[J]. Advances in Differential Equations, 2007, 11(4):399-418. 